\title{
NEMATODOS FITOPARÁSITOS ASOCIADOS AL CULTIVO DE TAGETES ERECTA EN EL DISTRITO VIRÚ, LA LIBERTAD, PERÚ
}

\section{PLANT- PARASITIC NEMATODES ASSOCIATED WITH CROP OF TAGETES ERECTA IN VIRU DISTRICT, LA LIBERTAD, PERU}

\author{
Santos Nélida Murga - Gutiérrez'
}

Fecha de recepción: 20 de mayo de 2006, fecha de aceptación: 24 de enero de 2007.

Forma de citar: Murga - Gutiérrez, SN. 2007. Nematodos fitoparásitos asociados al cultivo de Tagetes erecta en el Distrito Virú, La Libertad, Perú. Neotropical Helminthology, vol. I, no. I, p. 15-20.

\section{Resumen}

Se determinaron los géneros de nematodos fitoparásitos asociados al cultivo de Tagetes erecta L. "marigold" sembrado en el distrito Virú, departamento La Libertad, Perú, y sus frecuencias y densidades poblacionales. Entre marzo y diciembre de 2002, fueron colectadas al azar muestras de suelo de cultivo y de raíces de plantas de T. erecta en 14 parcelas sembradas con esta especie vegetal. Las muestras de suelo se procesaron mediante la técnica de Christie y Perry y las de raíces mediante la de Baermann modificada en bandeja; también se realizó coloración de raíces. Se encontraron diez géneros de nematodos fitoparásitos asociados al cultivo de $T$. erecta en el distrito Virú; los cuales, según frecuencia descendente de parcelas con cada género en el suelo fueron: Criconemoides $85,7 \%$, Helicotylenchus 78,6\%, Tylenchus 78,6 \%, Meloidogyne 71,4 \%, Hemicycliophora 64,3 \%, Aphelenchoides 57,1 \%, Trichodorus 35,7 \%, Paratylenchus 28,6 \%, Tylenchorhynchus 28,6 \% y Pratylenchus 21 , 4 \% y en las raíces: Meloidogyne 64,3 \%, Helicotylenchus 42,9 \% y Criconemoides 28,6\%. Los tres primeros presentaron las densidades poblacionales más altas. Meloidogyne fue el único encontrado dentro del tejido de algunas raíces noduladas, aunॄue mayormente como formas inmaduras y la producción de huevos fue nula o escasa.

Palabras clave: Nematodos fitoparásitos, Meloidogyne, Tagetes erecta, planta antagónica.

\section{Abstract}

The general, freeuency and population density of plant-parasitic nematodes associated with Tagetes erecta crops (marigold) in the District of Viru, La Libertad, Peru, were determined. Soil and root samples were collected between March and December 2002, from 14 planted fields. Nematodes were extracted from soil samples by the Christie and Perry's techniQue, and from root samples by the modified Baermann's technique. The roots were also stained for detection of endoparasitic nematodes. Ten genera of plant-parasitic nematodes were found associated with crop of $T$. erecta in Viru district, which in decreasing order of freeuency of fields with each genus extracted from soil were: Criconemoides 85,7 \%, Helicotylenchus 78,6\%, Tylenchus 78,6\%, Meloidogyne 71,4\%, Hemicycliophora 64,3 \%, Aphelenchoides 57,1 \%, Trichodorus 35,7 \%, Paratylenchus 28,6 \%. Tylenchorhynchus 28,6 \% and Pratylenchus 21,4 \% and those extracted from roots were: Meloidogyne 64,3\%, Helicotylenchus 42,9\% and Criconemoides 28,6\%. The first three genera showed the highest population densities. Meloidogyne spp. were found inside the tissue of some galls and mostly presented immature forms and null or scarce production of eggs.

Keywords: Plant-parasitic nematodes, Meloidogyne, Tagetes erecta, Antagonistic plant.

(I) Departamento de Microbiología y Parasitología, Facultad de Ciencias Biológicas, Universidad Nacional de Trujillo, Av. Juan Pablo II s/n, Trujillo, Perú. 


\section{INTRODUCCIÓN}

Tagetes erecta "marigold", también conocida en Perú como "flor de muerto", es una especie Que pertenece a la familia botánica Asteraceae, antes Compositae (Heyden, 2002), nativa de Centroamérica, y cultivada desde la época precolombina (Reynoso, 1999). En el mundo, este cultivo ha adQuirido gran importancia comercial, debido a la gran demanda Que tienen sus flores por ser una fuente rica de carotenoides y sus derivados xantofílicos, pigmentos naturales de color amarillo, utilizados en la elaboración de alimentos balanceados y también, como colorante para alimentos de consumo humano (Reynoso, 1999; Delgado, 1997; Flores \& Lazo, 1996).

En el Perú, T. erectaes cultivada para la comercialización de sus flores Que tienen gran demanda en el mercado nacional e internacional, lo cual constituye fuente de ingresos económicos para los agricultores Que los cultivan, así como, para Quienes lo industrializan y lo exportan. Este cultivo se ha incrementado en los últimos años, principalmente en los valles de Cañete, Lurín, Chancay, Barranca y Chosica (Departamento Lima) y en los valles de Virú y Chicama (Departamento La Libertad), Chira y Piura (Departamento Piura). En la Provincia Virú, se siembran mas de 1800 ha, según información obtenida en la Oficina de Información Agrícola del Ministerio de Agricultura, 2002. Entre los organismos patógenos Que podrían afectar el cultivo de "marigold" se han descrito principalmente a especies de hongos, insectos y ácaros (Reynoso, 1999), también puede ser afectado por virus, bacterias y nematodos.

T. erecta es una planta antagónica o planta repelente de nematodos, y generalmente, su cultivo está recomendado como medida para controlar algunos nematodos fitoparásitos (Winoto, 1969). Las plantas de "marigold" también pueden ser afectadas por algunas poblaciones de nematodos (Topp et al., 1998) observándose, en algunos casos, susceptibilidad a éstos. Los nematodos fitoparásitos ocasionan perjuicios directos o indirectos a las plantas, y el daño puede incrementarse cuando interactúan con otros patógenos (Nickle, 1991). Reduciendo la calidad y la cantidad de los productos del cultivo, asimismo, los nematodos pueden disminuir el tiempo de productividad de las plantas.

En la revisión bibliográfica, no se encontraron trabajos publicados sobre nematodos fitoparásitos asociados al cultivo de $T$. erecta en el Perú. Tampoco se encuentra investigaciones sobre nematodos asociados a cultivos de "marigold" en La Libertad. Por ello, es importante realizar estudios Que permitan conocer cuáles son los nematodos Que están asociados a esta especie vegetal, así como, conocer su potencial empleo como cultivo de rotación para controlar las poblaciones fitonematológicas.

El presente trabajo estuvo orientado a determinar los géneros de nematodos fitoparásitos asociados al cultivo de $T$. erecta L. sembrada en el distrito Virú, departamento de La Libertad, Perú, en el 2002, y también a determinar sus frecuencias y densidades poblacionales en las parcelas sembradas.

\section{MATERIAL Y MÉTODOS}

Material de estudio

Se trabajó con muestras de suelo de cultivo y de raíces de $T$. erecta sembrada en 14 parcelas del distrito Virú, de la provincia Virú.

Área de estudio

Los muestreos fueron realizados en parcelas cultivadas con T. erecta "marigold" en los sectores: El Carmelo, Santa Elena, Huancaquito, Huancaco, Santa María, El Socorro, Chequere y Saraque del distrito y provincia de Virú, departamento de La Libertad, en la costa norte del Perú (Fig. I).

La edad de los cultivos varió entre 1 y 6 meses. Durante el muestreo la temperatura varió entre $16^{\circ} \mathrm{C}$ y $29^{\circ} \mathrm{C}, y$ la humedad relativa entre 75 y $85 \%$.

Recolección, procesamiento de las muestras y determinación de los nematodos

Las muestras fueron colectadas entre marzo y diciembre de 2002, utilizando una palana recta, y en número de 50 muestras de suelo y de raíces por hectárea (Canto, 1985) en las 14 parcelas sembradas, mediante un muestreo aleatorio simple. Cada muestra se colocó en una bolsa de polietileno debidamente codificada y se transportó al Laboratorio de Helmintología del Departamento de Microbiología y Parasitología de la Universidad Nacional de Trujillo (Perú), para su procesamiento $y$ análisis. 


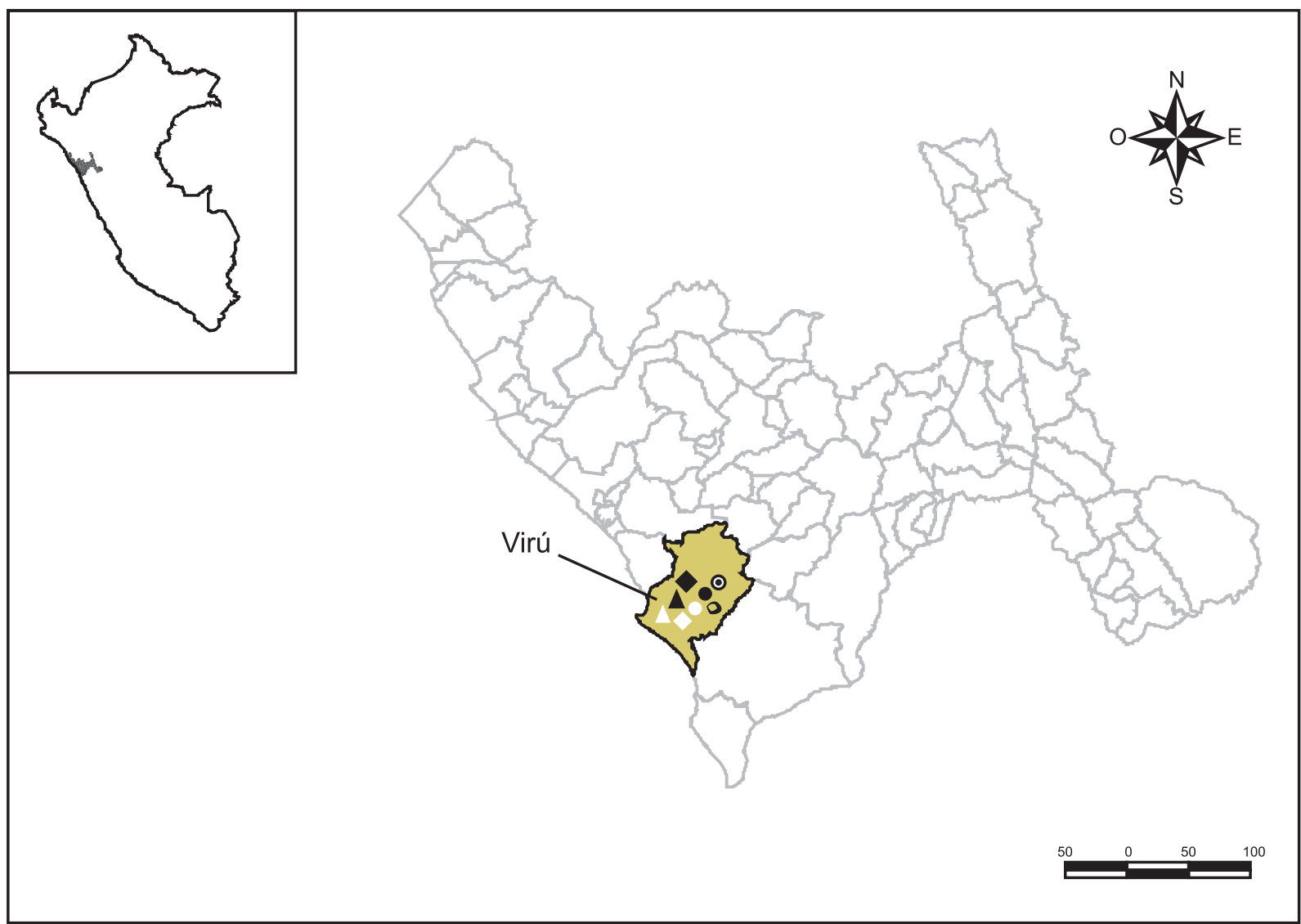

Figura 1. Ubicación geográfica del área de estudio en el Distrito y Provincia Virú, Departamento de La Libertad, Perú. Leyenda: El Carmelo $=\Delta$. Santa Elena $=\boldsymbol{\Delta}$. HuancaQuito $=\diamond$. Huancaco $=0$, Santa María $=\diamond$, El Socorro $=\bullet$, Chequere $=0$, Saraque $=\odot$.

Las muestras de suelo se procesaron mediante la técnica de Christie \& Perry, utilizando $100 \mathrm{~cm}^{3}$ de la muestra previamente homogeneizada (Oostenbrink, 1960), y las raíces se procesaron mediante la técnica de Baermann modificada en bandeja (Canto, 1985) utilizando $5 \mathrm{~g}$ de ellas. Cada muestra fue procesada por duplicado y luego de $48 \mathrm{~h}$ de reposo fueron extraídos los sedimentos, colocados en placas de Petri y examinados con ayuda de un microscopio invertido y uno compuesto. También se realizaron coloraciones de raíces con signos de daño (Zuckerman et al., 1985).

La determinación genérica de los nematodos se realizó por estudio de sus características morfológicas, empleando preparados en fresco, y en montaje previo aclaramiento de los helmintos por la técnica de Seinhorst (Zuckerman et al., 1985). Las características morfológicas observadas se compararon con las descripciones señaladas en la literatura correspondiente (Mai \& Lyon, 1960; S’Jacob \& Middelplaats, 1987).
La cantidad de parcelas con nematodos y sus densidades poblacionales promedio en las áreas muestreadas fueron expresadas en frecuencias relativas mediante la estadística descriptiva.

\section{RESULTADOS}

Se encontraron diez géneros de nematodos fitoparásitos asociados al cultivo de T. erecta en las parcelas examinadas del distrito Virú, La Libertad, Perú, los cuales, según su frecuencia de mayor a menor en las muestra de suelo fueron: Criconemoides, Helicotylenchus, Tylenchus, Meloidogyne, Hemicycliophora, Aphelenchoides, Trichodorus, Paratylenchus, Tylenchorhynchus y Pratylenchus, y en las raíces, Meloidogyne, Helicotylenchusy Criconemoides (Tabla I).

Las muestras de suelo presentaron entre 5 y 8 géneros de nematodos fitoparásitos y las muestras de raíces entre 0 y 2 géneros. El $100 \%$ de las parcelas estudiadas 
presentaron nematodos fitoparásitos en las muestras de suelo y el 71,4\%, en las muestras de raíces de $T$. erecta.

Las densidades poblacionales de los nematodos hallados variaron según el género de nematodo y según la parcela muestreada. Criconemoides, Helicotylenchus y Tylenchus, presentaron las densidades poblacionales más altas. En general, las densidades poblacionales de todos los nematodos encontrados fueron bajas, como se muestra en la Tabla 2.
Meloidogyne fue el único género encontrado dentro del tejido de algunas raíces examinadas, las cuales presentaban nódulos entre 1 y $3 \mathrm{~mm}$ de diámetro de tamaño. En los nódulos radiculares, principalmente estaban presentes las formas inmaduras del nematodo, con nula o escasa producción de huevos ( 0 a 2 huevos por $100 \mathrm{~g}$ de raíces con nódulos). Aunque la frecuencia de parcelas con Meloidogyne fue moderadamente alta, sus densidades poblacionales fueron bajas.

Tabla I. Frecuencia de parcelas con los géneros de nematodos fitoparásitos hallados en suelo de cultivo y en raíces de Tagetes erecta sembrada en 14 parcelas del distrito Virú, La Libertad, Perú, 2002.

\begin{tabular}{|c|c|c|}
\hline & \multicolumn{2}{|c|}{ Parcelas con nematodos (\%) } \\
\hline & Suelo & Agua \\
\hline Criconemoides & 85,7 & 28,6 \\
\hline Helicotylenchus & 78,6 & 42,9 \\
\hline Tylenchus & 78,6 & 0 \\
\hline Meloidogyne & 71,4 & 64,3 \\
\hline Hemicycliophora & 64,6 & 0 \\
\hline Aphelenchoides & 57,1 & 0 \\
\hline Trichodorus & 35,7 & 0 \\
\hline Paratylenchus & 28,6 & 0 \\
\hline Tylenchorhynchus & 28,6 & 0 \\
\hline Pratylenchus & 21,4 & 0 \\
\hline \multirow[t]{2}{*}{ Total } & 100,0 & 71,4 \\
\hline & \multicolumn{2}{|c|}{ Parcelas con nematodos (\%) } \\
\hline Géneros de nematodos & Suelo & Agua \\
\hline Criconemoides & $2-8$ & $2-3$ \\
\hline Helicotylenchus & $2-8$ & $1-3$ \\
\hline Tylenchus & $3-10$ & 0 \\
\hline Meloidogyne & $2-5$ & $1-3$ \\
\hline Hemicycliophora & $2-4$ & 0 \\
\hline Aphelenchoides & $2-5$ & 0 \\
\hline Trichodorus & $1-3$ & 0 \\
\hline Paratylenchus & $2-3$ & 0 \\
\hline Tylenchorhynchus & $3-5$ & 0 \\
\hline Pratylenchus & $2-3$ & 0 \\
\hline
\end{tabular}

\section{DISCUSIÓN}

Los géneros de nematodos fitoparásitos encontrados en las muestras de suelo y en las raíces de $T$. erecta en el área de cultivo evaluada del distrito y provincia de

Virú, La Libertad, presentaron características morfológicas coincidentes con aquellas descritas en la literatura consultada (Mai \& Lyon, 1960; S’ Jacob \& Middelplaats, 1987), lo cual permitió su determinación genérica. 
Los resultados obtenidos mostraron una amplia distribución de los nematodos hallados en el área de cultivo muestreada, y aunque algunos géneros se encontraron en una alta frecuencia de parcelas sembradas, en general, sus densidades poblacionales fueron bajas. Ello, probablemente debido a Que la mayoría de géneros encontrados comprenden a nematodos ectoparásitos, para los cuales la relación hospedero-parásito no siempre es permanente. Sin embargo, también podría ser Que el cultivo de $T$. erecta esté ejerciendo algún efecto antagónico sobre las poblaciones de algunos de los géneros hallados.

Los diez géneros hallados presentan especies Que son fitopatógenos potenciales de numerosos cultivos, a los cuales pueden afectar en la calidad y cantidad de sus cosechas. El hallazgo de Criconemoides, Helicotylenchus y Meloidogyne en suelo de cultivo y en raíces de "marigold" adouiere importancia significativa, porQue indicaría Que la relación hospedero-parásito es estrecha, por lo Que es necesario un estudio más profundo de estas asociaciones. Criconemoides y Helicotylenchus son ectoparásitos, con capacidad de producir daño a las plantas, y Meloidogyne es un endoparásito obligado y comúnmente perjudicial a los cultivos (Cepeda, 1989; Nickle, 1991). Se encontró dentro del tejido de algunos nódulos radiculares, aunque mayormente como formas inmaduras, y la producción de huevos fue nula o escasa. También se ha descrito la presencia de Meloidogyne en T. minuta, y con escasa capacidad de reproducción (Mc Sorley, 1999).

En relación a la alta frecuencia y la escasa densidad poblacional de Meloidogyne en las parcelas sembradas con $T$. erecta, los resultados expresan que las plantas de "marigold" no son buenos hospederos, y lo mismo sucedería con Pratylenchus, el menos frecuente en las parcelas y con baja densidad poblacional. Meloidogyne y Pratylenchus, son frecuentes en las parcelas de Virú cultivadas con otros vegetales, y en densidades poblacionales mayores a las encontradas en este estudio (Murga, 1988; Murga, 1997). Estos hallazgos reforzarían la posibilidad que $T$. erecta pueda tener un efecto perjudicial sobre las poblaciones de estos nematodos, si se emplea como rotación de cultivo.

Las plantas de diversas especies de Tagetes, incluida $T$. erecta, se comportan como plantas antagónicas o como plantas "repelentes", por ello, han sido utilizadas para controlar Meloidogyne spp., y sobre todo, Pratylenchus penetrans (Cepeda, 1989; Topp et al., 1998; Gapasin et al., 2006).

El cultivo intensivo de T. erecta o su manejo inadecuado podría alterar el comportamiento de esta especie como planta antagónica de estos nematodos. Los hallazgos en este estudio, han mostrado Que Meloidogyne penetra a las raíces, sin embargo, no se han encontrado formas maduras con una producción de huevos adecuada. Por lo Que, se confirma que T. erecta no es un buen hospedero de este género, ya Que los nematodos parecen ser incapaces de completar su ciclo de vida, y su capacidad de producir huevos es nula o escasa (McSorley, 1999).

Los resultados obtenidos indican la necesidad de ampliar los estudios a fin de obtener mayor conocimiento sobre la relación entre los nematodos fitoparásitos encontrados y el cultivo de T. erecta.

De acuerdo a los resultados hallados en este estudio el cultivo de T. erecta, podría ser utilizado como un cultivo de rotación en campos infestados con Meloidogyne y Pratylenchus. Esta medida tendría un efecto benéfico sobre las cosechas, siempre Que los agricultores manejen adecuadamente el cultivo de esta especie vegetal.

El presente estudio constituye el primer registro de géneros de nematodos fitoparásitos asociados al cultivo de $T$. erecta en el norte del Perú y en particular en el distrito Virú, perteneciente a la provincia Virú del departamento La Libertad.

\section{REFERENCIAS BIBLIOGRÁFICAS}

Canto, M. 1985. Nematología vegetal. Universidad Nacional Agraria, La Molina, Lima.

Cepeda, M. 1989. Nematología agrícola. Trillas, S.A. UAAAN. Saltillo, Coahuila.

Delgado, VG. 1997. Pigmentos de flor de cempasuchil (Tagetes erecta). Caracterización físico Química, procesamiento y eficiencia pigmentante (Tesis doctoral). Centro de Investigación y de Estudios Avanzados del Instituto Politécnico Nacional, Irapuato, Guanajuato.

Flores, E \& Lazo, P. 1996. Investigación tecnológica para la extracción del colorante de la flor de marigold y sus aplicaciones en la industria alimentaria. CICA - Universidad Católica de Santa María, 
Areeuipa.

Gapasin, RM, Ranchez, CV, Gergon, EB, Judal, ML, Pile, CV \& Miller, SA. 2002. Antagonistic plants for the management of rice root-knot nematode (Meloidogyne graminicola) in rice-onion system, consultado el 20 de diciembre de 2006, <http:// www.ag.vt.edu/IPMCRSP/presentations/Antagon istic\%20poster\%20US\%202003.pdf\#search =\% 22Antagonistic\%20Tagetes\%20spp\%22>

Heyden, D. 2002. Jardines botánicos prehispánicos. Revista Arqueología Mexicana, vol. X, pp. 1723.

Mai, WH \& Lyon, HH. 1960. Pictorial key of plantparasitic nematodes. 4ta. edn, Conwell University Press, London.

McSorley, R. 1999. Host suitability of potential cover crops for root-knot nematodes. Journal of Nematology, 31, (Supplement), 619-623.

Murga, SN. 1988. Nematodos parásitos en cítricos cultivados en Virú, Libro de Resúmenes del IX Congreso Nacional de Biología, Piura.

Murga, SN. 1997. Nematodos fitoparásitos asociados al cultivo de Asparagus officinales L. "espárrago" en la provincia de Virú. Perú, Libro de resúmenes de trabajos de investigación. Universidad Nacional de Trujillo, Trujillo.

Nickle, WR. 1991. Manual of agriculture nematology, Marcel Dekker, Inc, New York.

Oostenbrink, M. 1960. Estimating nematode populations by some selected methods, In IN Sasser \& WR lenkins (eds.), Nematology: Fundamentals and recent advances, The University of North Caroline Press, Chapel Hill.

Reynoso, E. 1999. Manual para el cultivo de flor de cempasuchil (Marigold). Bioquimex Reka, S.A. de C.V. México D.F.

S'lacob, II \& Middelplaats, WCT. 1987. Clave para identificación de los nematodos parásitos de las plantas, Universidad Agraria de Wageningen, Holanda en cooperación con la Universidad Nacional Agraria, La Molina, Lima.

Winoto, SR. 1969. Studies on the effect of Tagetes species on plant parasitic nematodes, $\mathrm{PhD}$ thesis, Agricultural University, Wageningen.

Topp, E, Millar, S, Bork, H \& Welsh, M. 1998. Effects of marigold (Tagetes sp.) roots on soil microorganisms. Biology and Fertility of Soils, vol. 27, pp. 149-154.

Zuckerman, BM, Mai, WF \& Harrison, MB. 1985. Plant nematology laboratory manual, University of Massachusetts Agricultural Experiment Station, Massachusetts.

\section{Autor para correspondencia / Author to} correspondence:

Santos Nélida Murga Gutiérrez

Departamento de Microbiología y Parasitología, Facultad de Ciencias Biológicas, Universidad Nacional de Trujillo, Av. Juan Pablo II s/n.

Trujillo, Perú

Correo electrónico/ E-mail: snmg100@yahoo.com

Telefax: (5 1)-44-251549.

\section{Anuncio/Anounce}

\section{Reunión Anual de la Organización de los Nematólogos de los Trópicos Americanos (ONTA)}

29 de octubre - 2 de noviembre de 2007, Córdova, Argentina

- Contacto:

Sitio web/ web site: http://www.ontaweb.org 